\title{
A Structure Parameter Design Method of DD Coils to Optimize Coupling Performance and Misalignment-tolerant Ability
}

\author{
Wei Zhang, Fusheng Wang, Juanjuan Guo, Longfeng Ye \\ School of Electrical and Automation Engineering, Hefei University of Technology, Hefei, 230009, China
}

\begin{abstract}
Aiming at the problems of weak coupling performance and poor misalignment-tolerant ability of the magnetic coupling structure of electric vehicle wireless charging system, this paper proposes a coil structure parameter design method. This paper analyzes the relationship between the system characteristics and the coupling coefficient of the wireless charging system based on the dual LCC resonant compensation network. Then, based on the analysis of the structure parameters of DD coils, a structure parameter design method to optimize coupling performance and misalignment-tolerant ability is proposed. Finally, the ANSYS MAXWELL 3-D finite element model of the DD magnetic coupling structure is built, and the validity and feasibility of the proposed design method are verified by simulation. Under the design method of coil structure parameter in this paper, the magnetic coupling structure can achieve excellent coupling performance and misalignment-tolerant ability.
\end{abstract}

\section{Introduction}

In recent years, with the increasingly serious global problems such as environmental pollution and energy crisis, people pay more and more attention to the development of renewable energy and new energy vehicle industry. With the advantages of environmental friendliness, convenience and intelligence, electric vehicles become more and more popular. Wireless power transmission is a kind of energy transmission method without direct electrical contact. Due to the advantages of flexible configuration, intelligent charging and safe operation, it has been more and more widely used in the fields of electric vehicles, medical equipment and household appliances[1]-[3].

Electric vehicle wireless charging systems require high-efficiency and high-power output capabilities. However, these are the core factors restricting the development of current wireless charging technologies. In order to improve the output power and system efficiency of electric vehicle wireless charging systems, it is essential to improve the coupling performance of the magnetic coupler. The current main method is to optimize the magnetic core structure and the coil shape.

In the current reference on the design of coil structure parameters for magnetic coupling structure, the University of Auckland proposes DD and DDQ coils on the basis of the circular coils. Their coupling performances and misalignment-tolerant abilities are significantly improved [4], literature [5] proposes a BP type coil, compared with DDQ coils, its wire consumption is reduced by about $20 \%$. Reference [6] proposes a DLDD type coil structure, which greatly improves the transmission distance and charging area. In reference [7], the spatial position of the coils is studied and optimized. Based on these references, this paper conducts a detailed study on the relationship between the coupling performance of the magnetic coupler and the structure parameters of DD coils, and gives a specific structure parameters design process.

This article analyzes the influence of the coupling coefficient on the output power and efficiency in the wireless charging system based on the dual LCC resonant network, and then introduces the parameters of the magnetic coupling structure. After that, the structure parameter design process of the DD magnetic coupling structure is proposed. Finally, on the basis of the proposed design method, the structure parameters of DD coils are obtained when the magnetic coupling structure has good coupling performance and misalignmenttolerant ability.

\section{System structure and analysis}

Since the magnetic coupler of the electric vehicle wireless charging system usually has an air gap of $150 \mathrm{~mm}-200 \mathrm{~mm}$ between the transmitting coil and the receiving coil, and is limited by the restricted chassis space of the electric vehicle, the leakage inductance between these coils is much greater than the mutual inductance, the coupling coefficient is usually lower than 0.3 . Therefore, it is necessary to add the resonant compensation network to improve the system power

*Corresponding author's e-mail: 497898752@qq.com 
transmission capability and efficiency. The basic resonant compensation circuit has four types: series-series (SS), series-parallel (SP), parallel-series (PS) and parallel-parallel (PP), when the above resonant network is used, the system characteristics such as the output power and efficiency are susceptible to the changes of the coupling coefficient and load. The uncertainty and instability of the system are more prominent. So scholars propose some high-order resonant compensation networks to improve the system performance, such as dual LC, dual LCL, LCC-S and dual LCC resonant compensation network, etc. Among these topologies, the dual LCC compensation structure has the characteristics of the unit power factor at the specific frequency and the output current is independent of the load, which is the popular compensation topology in current research [8]-[9].
The circuit structure of the wireless charging system based on the dual LCC resonant compensation network is shown in Figure 1. Among them, $U_{d}$ is the DC voltage source; $C_{d}$ is the input filter capacitor; $T_{1}-T_{4}$ form the high-frequency full-bridge inverter; the primary resonant compensation network is composed of the primary compensation inductor $L_{f l}$, the parallel compensation capacitor $C_{f l}$ and the series compensation capacitor $C_{l}$; $L_{1}$ and $L_{2}$ are the transmitting coil and the receiving coil respectively; the secondary compensation inductor $L_{f 2}$, the parallel compensation capacitor $C_{f 2}$ and the series compensation capacitor $C_{2}$ form the secondary resonant compensation network; $D_{l}-D_{4}$ form the full-bridge rectifier circuit; $C_{L}$ is the output filter capacitor. $M$ is the mutual inductance between the transmitting coil and the receiving coil.

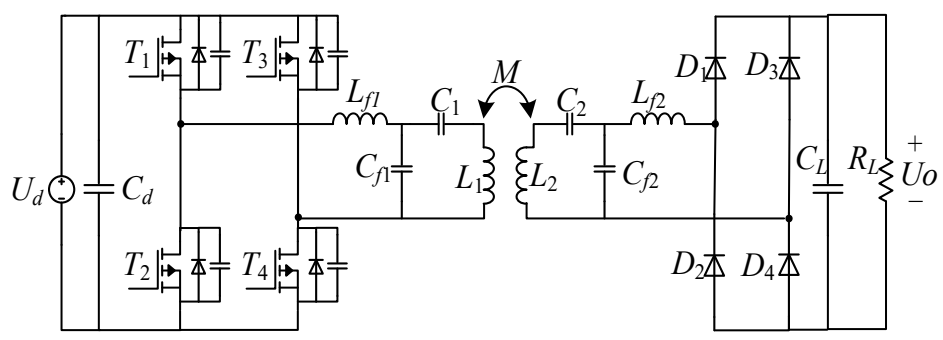

Figure 1. Wireless charging system based on the dual LCC resonant compensation network

Figure 2 shows the mutual inductance model of the resonant compensation system of the wireless charging system. The input DC source and full-bridge inverter circuit are equivalent to the AC source $u_{A B} ; i_{L f 1}$ and $i_{L f 2}$ are the input and output currents of the resonant compensation network, $i_{l}$ is the transmitting coil current, and $i_{2}$ is the receiving coil current. $Z_{\text {ref }}$ is the reflection impedance reflected from the secondary side to the primary side; the rectifier bridge and the battery load are equivalent to the resistance $R_{O S}$, and its voltage is $u_{O S}$.
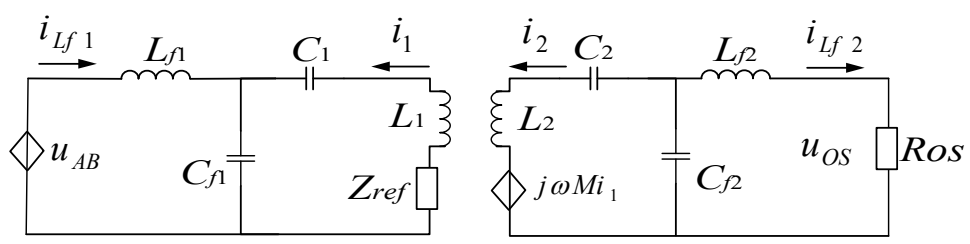

Figure 2. Dual LCC compensation topology mutual inductance model

The system output current is independent of the load when the system resonant frequency and compensation network parameters satisfy the following expressions:

$$
\left\{\begin{array}{l}
j \omega L_{f 1}+\frac{1}{j \omega \mathrm{C}_{f 1}}=0, j \omega L_{1}+\frac{1}{j \omega \mathrm{C}_{1}}+\frac{1}{j \omega \mathrm{C}_{f 1}}=0 \\
j \omega L_{2}+\frac{1}{j \omega \mathrm{C}_{2}}+\frac{1}{j \omega \mathrm{C}_{f 2}}=0, j \omega L_{f 2}+\frac{1}{j \omega \mathrm{C}_{f 2}}=0
\end{array}\right.
$$

The currents of the resonant network can be derived as:

$$
\left\{\begin{array}{l}
I_{L f 1}=\frac{M U_{O S}}{\omega L_{f 1} L_{f 2}}, \quad I_{1}=\frac{U_{A B}}{\omega L_{f 1}} \\
I_{L f 2}=\frac{M U_{A B}}{\omega L_{f 1} L_{f 2}}, \quad I_{2}=\frac{U_{O S}}{\omega L_{f 2}}
\end{array}\right.
$$

Where $U_{A B}$ and $U_{O S}$ are the RMS (Root Mean Square) values of the fundamental component of the input and output voltages $u_{A B}$ and $u_{O S}$ of the resonant compensation network, respectively, and the system output power is:

$$
P_{\text {out }}=\frac{M U_{A B} U_{O S}}{\omega L_{f 1} L_{f 2}}=\frac{8 k \sqrt{L_{1} L_{2}} U_{\mathrm{d}} U_{L}}{\pi^{2} \omega L_{f 1} L_{f 2}}
$$

It can be seen that the output power of the system is positively correlated with the coupling coefficient when the input and output voltages, and component parameters of the system are determined.

In the actual circuit, there are parasitic parameters in the coils and the compensation capacitors, and the winding loss is affected by the current flowing through it and the parasitic resistance. The parasitic resistance of inductor and capacitor can be calculated by the following formulas: 


$$
\begin{aligned}
& R_{1}=\frac{\omega L_{1}}{Q_{1}}, \quad R_{2}=\frac{\omega L_{2}}{Q_{2}} \\
& R_{f 1}=\frac{\omega L_{f 1}}{Q_{f 1}}, \quad R_{f 2}=\frac{\omega L_{f 2}}{Q_{f 2}} \\
& R_{C 1}=\frac{1}{D_{1} \omega C_{1}}, \quad R_{C 2}=\frac{1}{D_{2} \omega C_{2}} \\
& R_{C f 1}=\frac{1}{D_{C f 1} \omega C_{f 1}}, \quad R_{C f 2}=\frac{1}{D_{C f 2} \omega C_{f 2}}
\end{aligned}
$$

Where $Q$ and $D$ are the quality factor of the inductor and the loss factor of the capacitor, respectively.

According to the mutual inductance model shown in Figure 2, the resonant compensation system can be divided into a transmitting side circuit and a receiving side circuit. If only the fundamental loss is considered, the winding loss of the transmitting side circuit is:

$$
P_{\text {Tloss }}=I_{f 1}{ }^{2} R_{f 1}+I_{C f 1}{ }^{2} R_{C f 1}+I_{1}^{2}\left(R_{C 1}+R_{1}\right)
$$

The winding loss of the receiving circuit is:

$$
P_{\text {Rloss }}=I_{f 2}{ }^{2} R_{f 2}+I_{C f 2}{ }^{2} R_{C f 2}+I_{2}{ }^{2}\left(R_{2}+R_{C 2}\right)
$$

Among them, the RMS value of each branch current of expressions (5) and (6) is:

$$
\begin{aligned}
& I_{L f 1}=\mid \begin{array}{l}
j \omega L_{1}+\frac{1}{j \omega C_{1}}+\frac{1}{j \omega C_{f 1}}+R_{C f 1}+R_{C 1}+R_{1}+Z_{r f f} \\
\frac{1}{j \omega C_{f 1}}+R_{C f 1}
\end{array} I_{1} \\
& I_{C f 1}=\left|\frac{j \omega L_{1}+\frac{1}{j \omega C_{1}}+R_{C 1}+R_{1}+Z_{r f f}}{\frac{1}{j \omega C_{f 1}}+R_{C f 1}}\right| I_{1} \\
& I_{2}=\left|\frac{j \omega L_{f 2}+\frac{1}{j \omega C_{f 2}}+R_{C f 2}+R_{L f 2}+R_{O S}}{\frac{1}{j \omega C_{f 2}}+R_{C f 2}}\right| I_{L f 2} \\
& I_{C f 2}=\left|\frac{j \omega L_{f 2}+R_{L f 2}+R_{O S}}{\frac{1}{j \omega C_{f 2}}+R_{C f 2}}\right| I_{L f 2}
\end{aligned}
$$

The reflected impedance $Z_{\text {ref }}$ reflected from the secondary side to the primary side can be expressed as:

$$
\begin{aligned}
Z_{r e f} & =\frac{\omega^{2} M^{2}}{j \omega L_{2}+\frac{1}{j \omega C_{2}}+R_{C 2}+R_{2}+\left(\frac{1}{j \omega C_{f 2}}+R_{C f 2}\right) / /\left(j \omega L_{f 2}+R_{f 2}+R_{O S}\right)} \\
& =\frac{\omega^{2} \mathrm{k}^{2} L_{1} L_{2}}{j \omega L_{2}+\frac{1}{j \omega C_{2}}+R_{C 2}+R_{2}+\left(\frac{1}{j \omega C_{f 2}}+R_{C f 2}\right) / /\left(j \omega L_{f 2}+R_{f 2}+R_{O S}\right)}
\end{aligned}
$$

Therefore, the efficiency of the transmitting side circuit is:

$$
\eta_{p}=\frac{I_{1}^{2}\left[Z_{r e f}\right]}{I_{f 1}{ }^{2} R_{f 1}+I_{C f 1}{ }^{2} R_{C f 1}+I_{1}^{2}\left(R_{r e f}+R_{C 1}+R_{1}\right)}
$$

And the efficiency of the receiving side is:

$$
\eta_{s}=\frac{I_{f 2}{ }^{2} R_{O S}}{I_{f 2}{ }^{2} R_{f 2}+I_{C f 2}{ }^{2} R_{C f 2}+I_{2}{ }^{2}\left(R_{2}+R_{C 2}\right)+I_{f 2}{ }^{2} R_{O S}}
$$

The efficiency of the resonant compensation network is:

$$
\eta=\eta_{p} \eta_{s}
$$

It can be seen from equations (9) (10) (11) that the transmission efficiency of the resonant compensation network is related to the load, the parasitic parameters of the resonant compensation component and the coupling coefficient. In the wireless charging system, the load is generally fixed, and the parasitic parameters of the coil and capacitor are also small and can be controlled within a certain range. Compared with these factors, the coupling coefficient has a significant effect on the efficiency of the wireless charging system. The larger the coupling coefficient, the more the system efficiency can be improved.

\section{Structure parameter analysis and design method}

\subsection{Structure parameter analysis}

The transmitter and receiver of DD magnetic coupling structure are composed of coils (including the main coils and compensation coils), the $\mathrm{Mn}-\mathrm{Zn}$ ferrite with high permeability and the aluminum plate shielding layer.

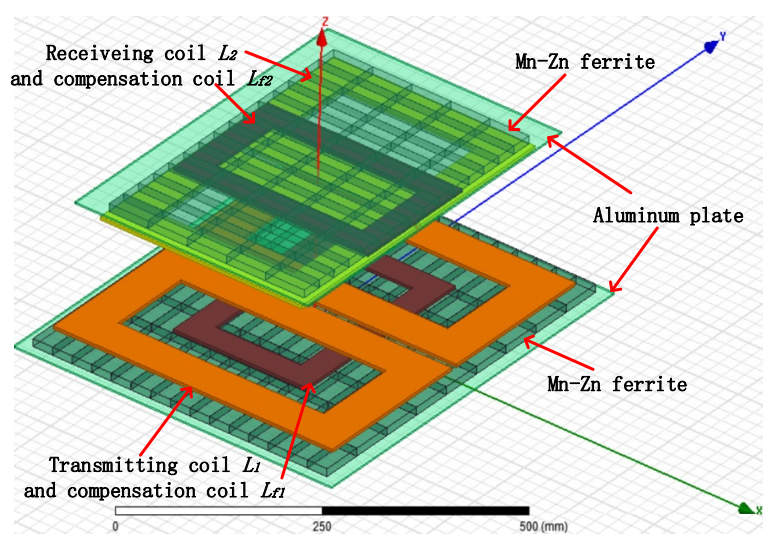

Figure 3. DD magnetic coupling structure

The following figure shows the structure of DD coils. The structure parameters are mainly composed of the external length, the external width, the internal length, the internal width, and the coil width.

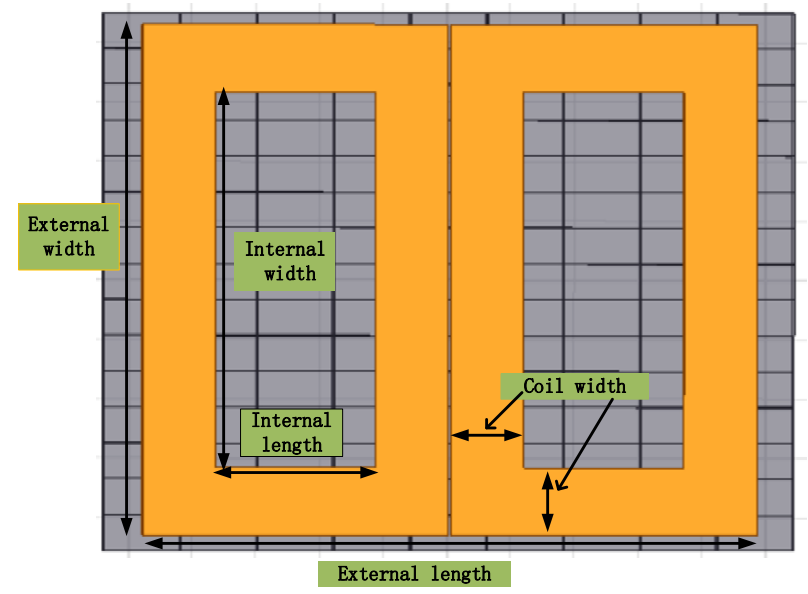

Figure 4. Main parameters of DD coils 
The external length and width of DD coils are determined by the internal sizes and the coil width. In addition, when the internal length and width are determined, the number of coil turns required to wind a certain inductance can also be uniquely determined. Therefore, the structure parameters of DD coils are determined by the internal size.

\subsection{Structure parameter design method}

When the internal lengths of the transmitting coil and the receiving coil are determined, DD coils with different structure parameters can be designed by changing their internal widths, that is, DD coils with different structure parameters can be designed when the two coils are combined with different length-width ratios. Similarly, when the length-width ratios of any two DD coils are the same, and the internal lengths of the transmitting and receiving coils are different, magnetic coupler of DD coils with different structure parameters are also designed. DD-type magnetic coupling structure with different structure parameters have different coupling coefficients. At the same time, the coupling coefficient will have different decrease trends if the receiving coil is misplaced, and the coil self-inductance will also have different degrees of variation.

When the transmitting coil and the receiving coil of the electric vehicle are misaligned, the coupling ability of the magnetic coupling structure will decrease, which will reduce the output power and efficiency of the wireless charging system. At the same time, the self-inductance of coils will change after the misalignment. If the variation is too large, it will affect the working state of the wireless charging system and bring unknown risks. Therefore, the misalignment-tolerant ability is also an important indicator to measure the performance of the magnetic coupling structure.

On the basis of the above analysis, with the goal of optimizing and improving the coupling performance and misalignment-tolerant ability of the DD magnetic coupler, this paper proposes the coil structure parameter design method shown in the following flow chart:

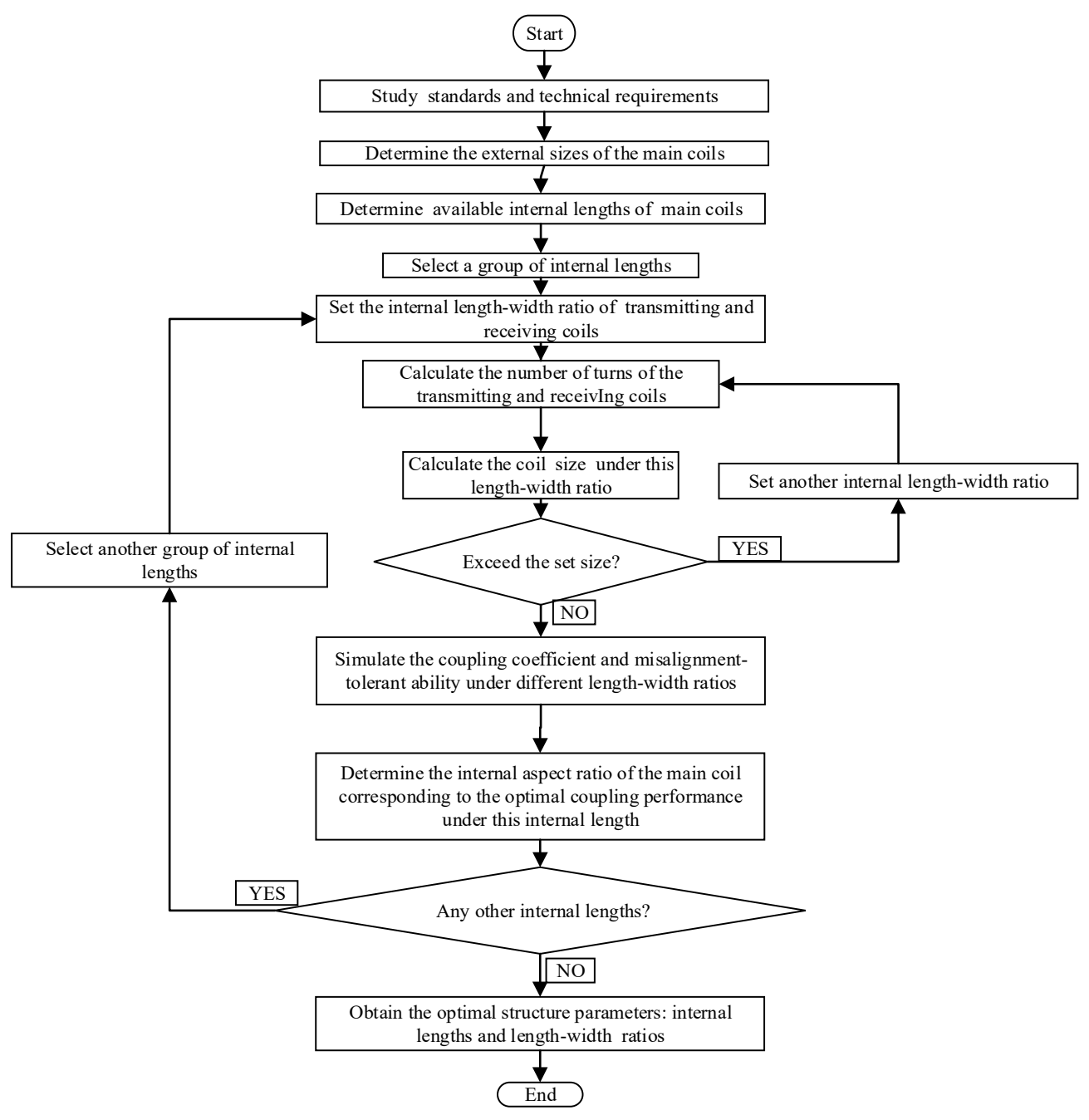

Figure 5. Flow chart of structure parameter design

In the above coil parameter design flow chart, the external sizes (length and width) of coils are calculated according to the standards and technical requirements of the electric vehicle wireless charging industry, followed by determining the available internal lengths of the transmitting coil and receiving coil, and then select a group of internal lengths and adjust their internal widths to adjust the internal length-width ratios, then calculate 
the number of coil turns needed to wind and judge whether the wound coil exceeds the size. If the requirements are satisfied, simulation experiments under different combinations of length-width ratios are performed, and then the receiver is misplaced to study the relationship between the variation of coil self-inductance and misalignment. Considering the coupling coefficient and the variation of the self-inductance comprehensively, the structure parameters of DD coils with the best coupling performance and misalignment-tolerant ability under this internal length are selected. After that, adjust the internal lengths of the transmitting coil and receiving coil, and repeat the above simulation process. After simulating the available internal lengths of the transmitting coil and the receiving coil, the structure parameters of DD coils with the best coupling performance and the misalignmenttolerant performance under the set standards and requirements can be obtained.

\section{Example design and simulation results}

Based on the above analysis and structure parameter design method, this section describes the above flow chart in detail with an example design.

The sizes and component parameters of the magnetic coupling structure to be designed are as follows:

Table 1. Main parameters of designed example

\begin{tabular}{cc}
\hline External sizes of transmitting coil & $\begin{array}{c}600 \mathrm{~mm} \times 450 \mathrm{~mm} \\
\text { (length } \times \text { width })\end{array}$ \\
\hline External sizes of receiving coil & $400 \mathrm{~mm} \times 300 \mathrm{~mm}$ \\
\hline
\end{tabular}

\begin{tabular}{cc}
\hline & (length $\times$ width) \\
\hline Transmission distance & $150 \mathrm{~mm}$ \\
\hline Working frequency & $85 \mathrm{kHz}$ \\
\hline $\begin{array}{c}\text { Self-inductance of transmitting } \\
\text { coil } L_{1}\end{array}$ & $360 \mu \mathrm{H}$ \\
\hline $\begin{array}{c}\text { Self-inductance of receiving coil } \\
L_{2}\end{array}$ & $240 \mu \mathrm{H}$ \\
\hline $\begin{array}{c}\text { Self-inductance of compensation } \\
\text { coil } L_{f 1} L_{f 2}\end{array}$ & $60.3 \mu \mathrm{H}$ \\
\hline
\end{tabular}

According to the standards and requirements, the ranges of the internal length of the transmitting coil and receiving coil that can be selected are $68-170 \mathrm{~mm}$ and $35-88 \mathrm{~mm}$, respectively. Within these ranges, the internal lengths of $170 \mathrm{~mm}$ and $88 \mathrm{~mm}$ are selected as examples for the following description. Then adjust the internal widths of the transmitting (receiving) coil to make the internal length-width ratio of the transmitting (receiving) coil is $0.5,0.75,1.0,1.25,1.5$ (the interval can also be further reduced). After that, determine the number of turns required for the transmitting (receiving) coil under the above length-width ratios, and verify whether the external sizes meet the set requirements. If it is not satisfied, the length-width ratio is not considered. If it meets the requirements, the next step can be carried out. Select the internal length-width ratio of the transmitting coil as 0.5 , and simulate the coupling coefficient when the internal length-width ratio of the receiving coil is 0.5 , $0.75,1.0,1.25$, and 1.5; then change the length-width ratio of the transmitting coil as $0.75,1.0,1.25,1.5$, and repeat the above process. Under the internal lengths of $170 \mathrm{~mm}$ and $88 \mathrm{~mm}$, the coupling coefficients under the above internal length-width ratios are shown in figure 6 .

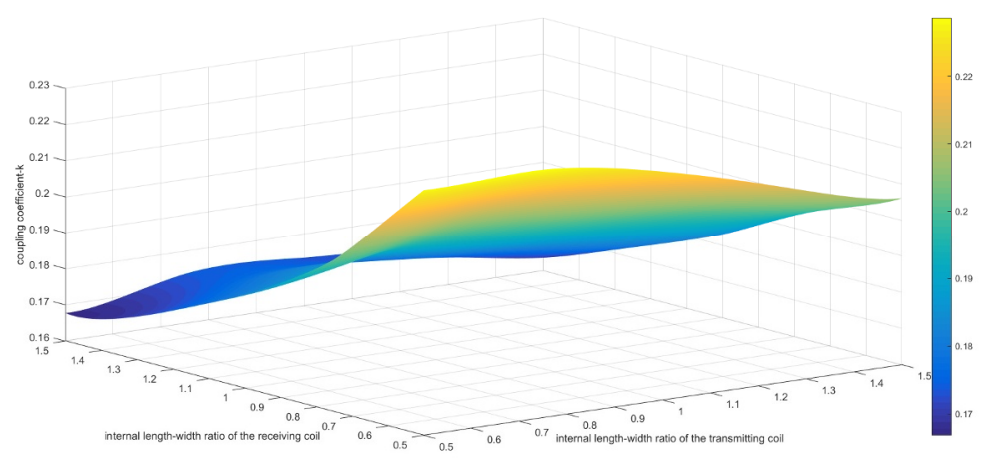

Figure 6. Coupling coefficients under different internal length-width ratios when fully aligned

It can be seen that when the internal lengths of the transmitting and receiving coils are determined, the smaller the internal length-width ratio (i.e., the larger the internal width), the higher the coupling coefficient of the magnetic coupling structure. However, in order to obtain the best coupling performance, it is necessary to study the coupling coefficient of the magnetic coupling structure when the internal lengths of the transmitting and receiving coils change.

In actual operation, misalignment between the transmitting coil and the receiving coil may occur. The coupling coefficient of the magnetic coupling structure will decrease and the coil self-inductance will fluctuate. Therefore, the misalignment-tolerant performance is also an important factor to evaluate the performance of magnetic coupling structure. When the wireless charging system is working, the possibility of misalignment in $y$ direction is higher than that in $\mathrm{x}$ direction. At the same time, in the $\mathrm{x}, \mathrm{y}$ direction, the misalignment is basically allowed to be within $50 \%$ of the external size of the receiving coil. Combined with the actual situation, this paper simulates and analyzes the misalignment-tolerant ability when the receiving coil moves $30 \%$ of the given external length (i.e. $400 \mathrm{~mm}$ ) in the y direction. 
As can be seen from Figure 7, when the receiving coil is moved by $120 \mathrm{~mm}$ in the y direction, the retention of the coupling coefficient under these above length-width ratios are basically the same, which is about $52.5 \%-53.6 \%$ of that when the magnetic coupling structure is fully aligned. In terms of the variation of coil self-inductance, when the receiving end moves $120 \mathrm{~mm}$, the self-inductances of the compensation coils $\left(L_{f 1}\right.$ and $\left.L_{f 2}\right)$ are basically unchanged; the retention of the self-inductance of the transmitting coil is smaller than that of the receiving coil, that is, the self-inductance of the transmitting coil is more sensitive to the misalignment; when the internal length-width ratio of the transmitting (receiving) coil is set to a certain value, the retention percentages of $L_{1}$ and $L_{2}$ self-inductance increase firstly and then decrease as the internal length-width ratio of the receiving (transmitting) coil increases. Therefore, there is an optimal internal length-width ratio which makes the coil have the strongest misalignment-tolerant ability.

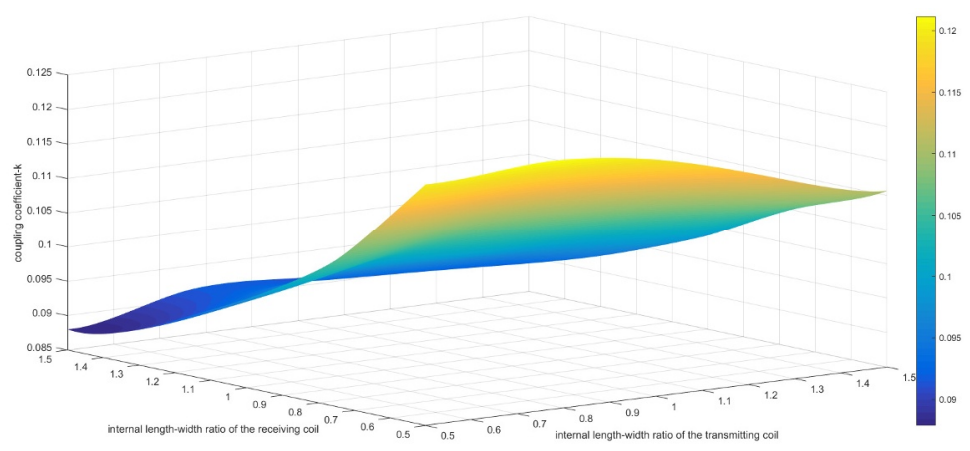

(a)

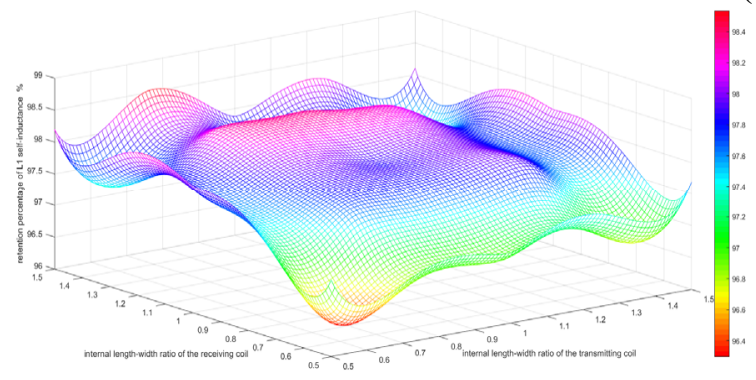

(b)

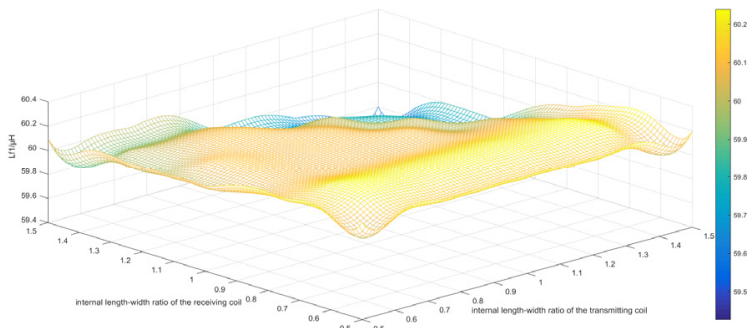

(d)

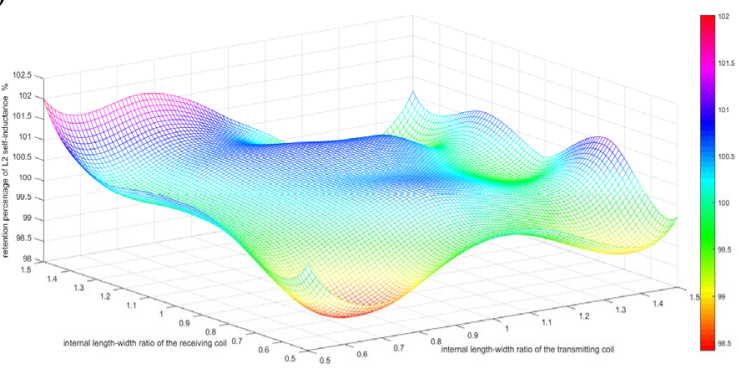

(c)

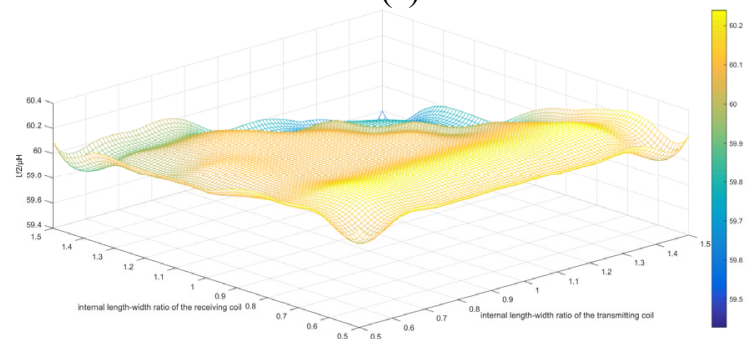

(e)

Figure 7. Misalignment-tolerant ability after 30\% misalignment (a) coupling coefficient (b) retention of $L_{1}$ (c) retention of $L_{2} \quad(\mathrm{~d})$ self-inductance of $L_{f 1}$

(e) self-inductance of $L_{f 2}$

Then, change the internal lengths of the transmitting coil and receiving coil, adjust their internal widths, and simulate the coupling coefficient and misalignment-tolerant ability of these coils under different combinations of internal length-width ratios. After simulating the available internal lengths of the transmitting coil and the receiving coil, considering the coupling coefficient of alignment and the misalignment-tolerant ability, the structure parameters of DD coils with the optimal coupling performance are obtained.

Under the set external size of the magnetic coupling structure, combined with the proposed coil structure parameter design method, the internal lengths of the transmitting and receiving coils designed in this paper are $165 \mathrm{~mm}$ and $80 \mathrm{~mm}$, the internal length-width ratios are 0.75 and 0.625 , respectively, and the coupling coefficient is 0.218 . When the receiving coil is moved by $30 \%$ in the y direction, the coupling coefficient is 0.115 , the self-inductance retention percentages of the transmitting coil and the receiving coil are $97.8 \%$ and $99.42 \%$, and the self-inductances of the compensation coils $L_{f 1}$ and $L_{f 2}$ are $60 \mu \mathrm{H}$ and $59.8 \mu \mathrm{H}$, respectively.

\section{Conclusion}

In order to achieve high power output and high efficiency operation of electric vehicle wireless charging systems, this paper studies the coil structure parameters of DD magnetic coupling structure, and proposes a design 
method of DD coils structure parameters based on coupling performance and misalignment-tolerant ability. The structure parameters of the transmitting coil and receiving coil under the given external sizes are designed. The 3-D finite element method simulation results verify that the magnetic coupling structure achieves the strongest coupling performance and misalignmenttolerant ability under this structure parameter, which helps to further improve the transmission power and efficiency of the wireless charging system.

The structure parameter design method of DD coils proposed in this paper can not only be used to design DD coils with strong coupling performance and misalignment-tolerant ability, but also can provide reference for the structure design of DDQ coils and BP coils. In the future, we will use the design method proposed in this paper as a basis, combined with the power density, system efficiency, magnetic field and biological safety, and cost to perform the multi-objective optimization of DD coils structure parameter.

\section{References}

1. F. Lu, H. Zhang, Heath H., Chris Mi. (2017) A dual-coupled LCC-compensated IPT system to improve misalignment performance[C]. 2017 IEEE PELS Workshop on Emerging Technologies: Wireless Power Transfer (WoW), 1-8.

2. Q. Ke, W. Luo, G. Yan, et al. (2016) Analytical Model and Optimized Design of Power Transmitting Coil for Inductively Coupled Endoscope Robot[J]. IEEE Transactions on Biomedical Engineering, 63(4): 694-706.

3. Z. Wang, Y. Li, Y. Sun, et al. (2013) Load detection model of voltage-fed inductive power transfer system[J]. IEEE Transactions Power Electronics, 28(11): 5233-5243.

4. Budhia M, Boys J T, Covic G A, et al. (2013) Development of a single-sided flux magnetic coupler for electric vehicle IPT charging systems [J]. IEEE Transactions on Industrial Electronics, 60(1): 318-328.

5. Covic G A, Kissin M L G, Kacprzak D, et al. (2011) A bipolar primary pad topology for EV stationary charging and highway power by inductive coupling[C]//EEE Energy Conversion Congress and Exposition (ECCE), Phoenix, 1832-1838.

6. Z. Wang, C. Hu, Y. Sun, X. Dai, (2015) Design of Magnetic Coupler for Inductive Power Transfer System Based on Output Power and Efficiency. Transactions of China Electrotechnical Society, 30(19):26-31.

7. C. Xia, Y. Sun, N. Jia, et al. (2012) Magnetic Circuit Parameter Optimization for Coupled Magnetic Resonance Power Transfer System [J]. Transactions of China Electrotechnical Society, 27(11):139-145.

8. L. Yuan, B. Li, Y. Zhang. et al. (2015) Maximum efficiency point tracking of the wireless power transfer system for the battery charging in electric vehicles[C]//18th International Conference on Electrical Machines and Systems (ICEMS), 11011107.

9. Li H L, Hu A P, Covic G A. (2012) A direct AC-AC converter for inductive power-transfer systems [J]. IEEE Transactions on Power Electronics, 27(2): 661-668 